\title{
Coherent current transport in wide ballistic Josephson junctions
}

\author{
P. Samuelsson ${ }^{a}, \AA$ A. Ingerman ${ }^{2}$ and G. Johansson ${ }^{c}$, E.V. Bezuglyi ${ }^{d}$, V.S. Shumeiko ${ }^{b}$, and G. Wendin ${ }^{b}$ \\ ${ }^{a}$ Département de Physique Theorique, Université de Genève, CH-1211 Genéve 4, Switzerland. \\ ${ }^{b}$ Department of Microtechnology and Nanoscience, \\ Chalmers University of Technology, S-41296 Göteborg, Sweden. \\ ${ }^{c}$ Institut für Theoretische Festkörperphysik, Universität Karlsruhe, 76128 Karlsruhe, Germany. \\ ${ }^{d}$ B. Verkin Institute for Low Temperature Physics and Engineering, Kharkov 61164, Ukraine. \\ R. Kürsten, A. Richte1 $\dagger$ and T. Matsuyama and U. Merkt \\ Institut für Angewandte Physik, Universität Hamburg, Jungiusstrasse 11, D-20355, Germany.
}

\begin{abstract}
We present an experimental and theoretical investigation of coherent current transport in wide ballistic superconductor-two dimensional electron gas-superconductor junctions. It is found experimentally that upon increasing the junction length, the subharmonic gap structure in the currentvoltage characteristics is shifted to lower voltages, and the excess current at voltages much larger than the superconducting gap decreases. Applying a theory of coherent multiple Andreev reflection, we show that these observations can be explained in terms of transport through Andreev resonances.
\end{abstract}

PACS numbers: $74.45 .+\mathrm{c}, 74.50 .+\mathrm{r}, 73.23 . \mathrm{Ad}$

Ballistic superconducting junctions are of great interest for studying fundamental properties of coherent electronic transport. The concept of Josephson effect in superconductor-normal metal-superconductor junctions was originally formulated for ballistic junctions [1]. However, until recently, experiments could only be performed on metallic diffusive junctions, which has limited the range of phenomena observed. Experimental realization of the ballistic regime became possible when a highmobility two-dimensional electron gas (2DEG) was employed to connect superconducting (S) electrodes [2]. The recent experimental interest [3] has been focused on junctions with InAs 2DEGs which form highly transparent 2DEG-S interfaces with large probability of Andreev reflection. Moreover, electrostatic gating 2, 44 has made it possible to control junction parameters.

The dc Josephson effect in ballistic S-2DEG-S junctions has been extensively studied both experimentally $[2,3,[4,[5,6]$ and theoretically [7]. Less investigated are the transport properties of the junctions in the presence of a voltage bias between the superconductors, the regime of multiple Andreev reflection (MAR) [8]. In particular, a conclusive experimental picture of the subharmonic gap structure (SGS) in the current-voltage characteristics is lacking. Moreover, the main tool for analyzing the experimentally observed current-voltage characteristics has been the theory of Octavio et al. [9] (OTBK). However, this is not appropriate for junctions which show a dc Josephson effect, i.e. which are in the coherent transport regime, since the OTBK theory is restricted to incoherent MAR transport.

The theory for coherent MAR transport in junctions much shorter than the superconducting coherence length [10] has with great accuracy explained experiments with atomic point contacts [1], both the details of the SGS at $e V=2 \Delta / n$ and the magnitude of the excess current at voltages much larger than the superconducting gap. This theory however cannot be directly applied to the experimentally studied junctions [2, 3, 4, 5, 6], generally of lengths $L$ of the order of the superconducting coherence length $\xi_{0}$.

In this letter we report a systematic experimental investigation of coherent current transport in ballistic NbInAs-Nb juctions with 2DEG-S interfaces of high transparency. For every junction we investigated the three key transport characteristics: SGS, excess current, and critical Josephson current. All investigated junctions show a dc Josephson effect. Our main experimental observation is that the SGS is shifted down in voltage upon increasing the distance between the superconducting electrodes, i.e. the junction length. Moreover, the excess current is found to decrease with increasing junction length.

These observations can be explained within a coherent, multimode MAR-theory. The shift of the SGS results from transport through Andreev resonances 12, 13): the Andreev resonances, closely related to the Andreev levels carrying the dc Josephson current, are shifted to lower energies upon increasing the junction length, consequently shifting the SGS down in voltage. Similarly, the excess current decreases with increasing junction length for the experimentally relevant junction lengths, $L \lesssim \xi_{0}$.

A schematic picture of the junction is shown in Fig. 1 Two niobium electrodes are deposited directly on the 2DEG which forms spontaneously at the surface of single crystal InAs [14]. In the following step, the junction is covered by a $\mathrm{SiO}_{2}$ layer that provides insulation to the $\mathrm{Al}$ gate electrode grown on top. On three different chips $\mathrm{A}, \mathrm{B}, \mathrm{C}$, junctions of five different interelectrode distances $L=105(\mathrm{~A}), 115(\mathrm{~B}), 145(\mathrm{~B}), 160(\mathrm{~A})$ and $200(\mathrm{C}) \mathrm{nm}$ have been fabricated. All junctions have a width $W=50 \mu \mathrm{m}$. 
The sheet carrier density $n_{\mathrm{s}}$, the effective mass $m^{*}$ and the mobility $\mu$ are controlled with the top gate to give the largest 2DEG-conductance. Typical parameters for the unprocessed InAs are $n_{\mathrm{s}}=2 \times 10^{16} \mathrm{~m}^{-2}$ and $m^{*}=$ $0.033 m_{\mathrm{e}}$, where $m_{\mathrm{e}}$ is the free electron mass. This gives a Fermi wave length $\lambda_{\mathrm{F}}=18 \mathrm{~nm}$ and $v_{\mathrm{F}}=1.2 \times 10^{6} \mathrm{~ms}^{-1}$. The mobility was not measured on the actual samples, but is typically of order $\mu=10000 \mathrm{~cm}^{2}(\mathrm{Vs})^{-1}$. The measured critical temperature of $\mathrm{Nb} T_{\mathrm{c}}=9.0 \mathrm{~K}$ corresponds to a superconducting gap $\Delta=1.4 \mathrm{meV}$ close to the bulk value. The superconducting coherence length is thus $\xi_{0}=\hbar v_{\mathrm{F}} / \Delta=600 \mathrm{~nm}$. The normal resistance $R_{\mathrm{N}}$
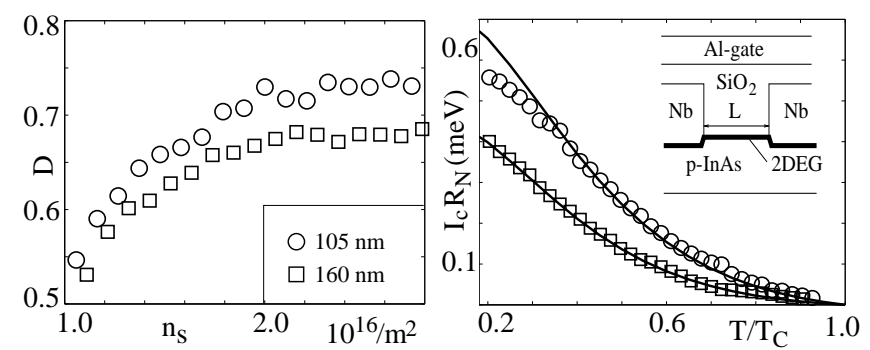

FIG. 1: Left: Average normal transparency $D=R_{\mathrm{N}} / R_{\mathrm{Sh}}$ as a function of $n_{\mathrm{s}}$ for samples of lengths $L=105 \mathrm{~nm}$ and $160 \mathrm{~nm}$. Right: $I_{\mathrm{c}} R_{\mathrm{N}}$ product as a function of $T$ for the same samples. The thin lines are the theory, with $I_{\mathrm{c}} R_{\mathrm{N}}(T=0)$ scaled for best fit by $1.65(L=105 \mathrm{~nm})$ and $2.4(L=160 \mathrm{~nm})$ and $\hbar v_{\mathrm{F}} / E_{\mathrm{A}}=0.54 \xi_{0}$ (see text). Inset: A schematic cut through the junction, displaying the kink in the 2DEG.

was measured at a voltage $e V \gg \Delta$. The ratio between the Sharvin resistance $R_{\mathrm{Sh}}=h /\left(2 e^{2}\right)\left[\lambda_{\mathrm{F}} / 2 W\right]$ and the normal resistance, i.e. the average transmission probability per conduction mode, is $D \sim 0.7$ for $n_{\mathrm{s}}>2 \times 10^{16}$ $\mathrm{m}^{-2}$ (see Fig. 1). The interfaces between the 2DEG and the metal electrodes are thus highly transparent. Attributing the small difference in resistance between the 105 and $160 \mathrm{~nm}$ junction to impurity scattering gives a mean free path $l \approx 300 \mathrm{~nm}$. This demonstrates that the transport is in the ballistic regime $(L<l)$. All junctions show a dc Josephson current, with $I_{\mathrm{c}} R_{\mathrm{N}}$ products $0.6,0.55,0.52,0.45$ at $1.8 \mathrm{~K}$ monotonically decreasing with $L$ from $L=105$ to $200 \mathrm{~nm}$. The temperature dependence of the dc Josephson current is shown in Fig. 1 for two junctions $L=105$ and $160 \mathrm{~nm}$.

In Fig. 2] the experimentally measured differential resistance for the different junctions is depicted. For all junctions, the resistance shows SGS at voltages $\mathrm{eV}<2 \Delta$. As an overall tendency, the SGS is shifted to lower voltages when increasing junction length. For junctions on the same chip this holds strictly. All SGS-features are symmetric around $V=0$ and scale roughly with temperature like the gap $\Delta(T)$, up to $T_{\mathrm{c}}$.

A decrease in the excess current as a function of length $L$ is also observed in the experiment. The $I_{\text {exc }} R_{\mathrm{N}}$ product for the different junctions is shown in Fig. 4 below, displaying an overall decrease with increasing $L$. This holds strictly, just as the shift of the SGS, for junctions on the same chip. The systematic shift of the SGS and the decrease of the excess current with junction length are the main experimental observations in this work.

Based on these findings, we model the junction as a ballistic, two-dimensional normal conductor of length $L$ and width $W$ connected to two bulk superconducting electrodes via highly transparent interfaces. The normal reflection in the junction is assumed to be concentrated to the kink in the 2DEG formed during the junction processing, see Fig. 11 For simplicity, the normal reflection is taken to be specular, reducing the problem to summing over independent modes. The width of the junction $W \gg \lambda_{F}$, giving a number of transport modes $2 W / \lambda_{F}=5600$. No sources of decoherence, e.g. inelastic scattering [15], are taken into account in the model.

We perform our theoretical analysis within the scattering approach to the Bogoliubov-de Gennes equation 16]. This allows us to express the dc Josephson current 17 as well as the current in voltage biased junctions 12] in terms of the transmission and reflection amplitudes $t_{m}(E)$ and $r_{m}(E)$ of each transport mode $m$ and the mode independent Andreev reflection probability $\alpha(E)$ at the 2DEG-S interface. The scattering amplitudes are given by $r_{m}=i \sqrt{R}\left(1+q_{m}^{2}\right) /\left(1+R q_{m}^{2}\right)$ and $t_{m}=q_{m}(1-R) /\left(1+R q_{m}^{2}\right)$ where $q_{m}=\exp \left[i k_{m} L\right]$; $k_{m}(E)=\left[2 m^{*} / \hbar^{2}\left(E_{\mathrm{F}}-E_{m}+E\right)\right]^{1 / 2}$, where $E_{\mathrm{F}}$ is the Fermi energy and $E_{m}$ is the transverse mode energy. The reflection probability of each interface $R$, taken to be mode independent, is given from the averaged total transmission probability $D$ as $R=(1-D) /(1+D)$, giving $R=0.16$ for $D=0.72$ ( $D$ for the $105 \mathrm{~nm}$ junction at $n_{\mathrm{s}}=2 \times 10^{16} \mathrm{~m}^{-2}$ ).

The Andreev reflection amplitude at the 2DEG-S interface is given by $\alpha(E)=\exp [-i \arccos (E / \Delta)]$. It is however not possible to apriori conclude that quasiparticles are Andreev reflected directly at the 2DEG-S interface near the kink, they might spend a certain time $\hbar / E_{\mathrm{A}}$ underneath the superconductor [18]. This can be incorporated into the model in the simplest possible way by multiplying $\alpha(E)$ with a phase factor $\exp \left(i E / E_{\mathrm{A}}\right)$, where $E_{\mathrm{A}}$ has to be determined by comparison to the experimental data. 19]

The total dc-current can be written as a sum of the currents for the individual modes [20], i.e. $I=\sum_{m} I_{m}$. In the general case, it is not possible to analytically calculate the current for arbitrary voltage, but one has to resort to numerics. It is important to note that most transport modes $m$ have effective lengths $L / \sqrt{1-(m / N)^{2}}$ close to the physical length $L$. Thus, the sharp features in the SGS predicted in single mode junctions of finite length 13. are not washed out but are merely broadened by the summation over the modes.

The qualitative behavior of the SGS as a function of junction length is clear from the numerics in Fig. 3] One 

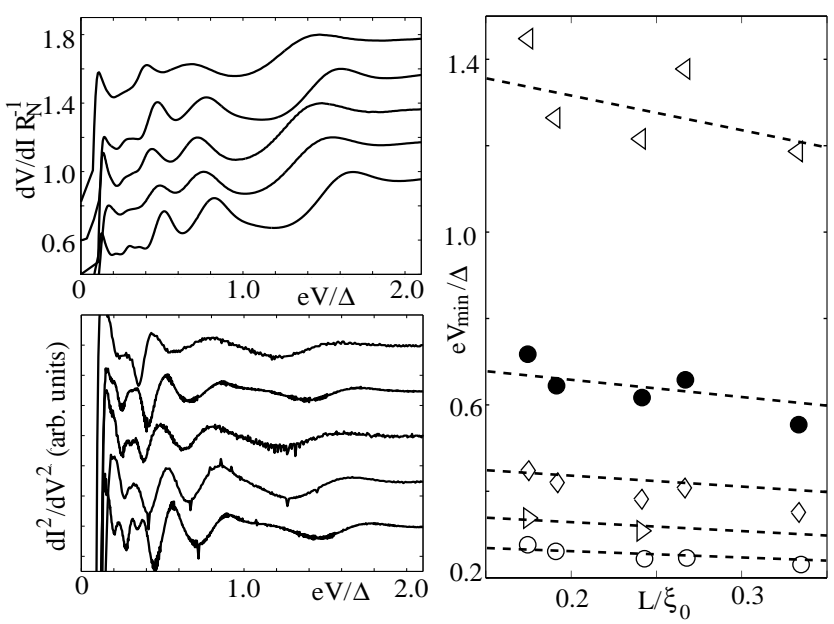

FIG. 2: Left: Measured differential resistances $d V / d I$ (upper) and (numerically differentiated) $d^{2} I / d V^{2}$ (lower) as a function of $V$ for $T=1.8 \mathrm{~K}$. The junction lengths are from bottom to top, $L=105,115,145,160$ and $200 \mathrm{~nm}$. The traces have been successively shifted for clarity. Right: Positions of the minima $V_{\min }$ of the $d^{2} I / d V^{2}$ (lower left) for the SGS $n=1$ $(\triangleleft), n=2(\bullet), n=3(\diamond), n=4(\triangleright$, only two minima clearly visible) and $n=5(\circ)$. Dashed lines are given by Eq. (1), with an additional length $\hbar v_{\mathrm{F}} / E_{\mathrm{A}}=0.6 \xi_{0}$.

sees how the SGS are shifted down to lower voltages when increasing the junction length $L$. The nature of this effect is the shift of broadened MAR resonances [12, 13. The MAR resonances are closely related to the Andreev levels carrying the dc Josephson current. The Andreev levels are shifted towards lower energies upon increasing the junction length, leading to a corresponding shift in the SGS. Additional numerics shows that the general tendency of a shift of the SGS towards lower voltages is independent of the reflection probability $R$, while the details of the SGS depend on $R$. For perfectly transparent 2DEG-S interfaces, however, the SGS are absent for $k T \ll \Delta[8]$ for any junction length [13].

Quantitatively, we have performed a detailed numerical investigation of the SGS $(n=1-5)$ in the experimentally relevant limit of small normal reflectivity, $R \leq 0.16$ and small effective junction length, $L_{\text {eff }}<\xi_{0}$. The effective length $L_{\mathrm{eff}}=L+\hbar v_{\mathrm{F}} / E_{\mathrm{A}}$ is the sum of the geometric length of the junction $L$ and the distance $\hbar v_{\mathrm{F}} / E_{\mathrm{A}}$ the quasiparticles propagate under the superconductors before they are Andreev reflected. The analysis of the numerical results shows that close to $V=2 \Delta /$ en the second derivative 21] $d^{2} I / d V^{2}$ has minima which depend on junction length as

$$
V_{\min }=\frac{2 \Delta}{e n}\left(1-0.4 \frac{L_{\text {eff }}}{\xi_{0}}\right), \quad n=1,2, . .
$$

for $L_{\text {eff }}<0.4 \xi_{0}$ for all $n$ (for $n>1$, it holds for even longer $\left.L_{\text {eff }}\right)$. This length dependence is in strong contrast to the result of the theory of incoherent MAR [9], which

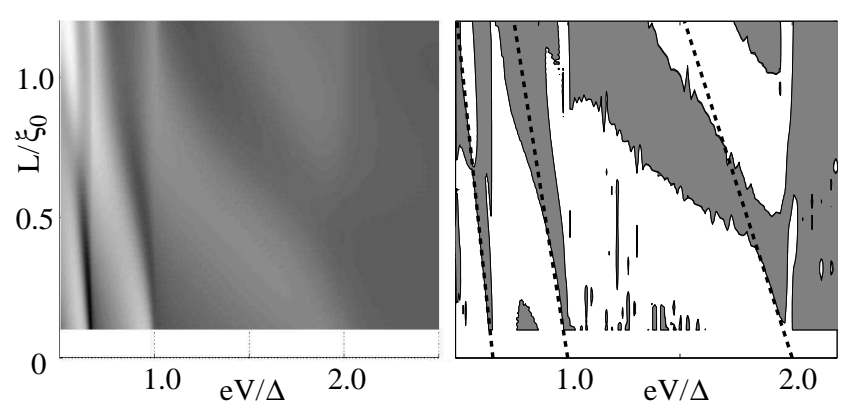

FIG. 3: Left: Plot of the numerically calculated $d I / d V$ as function of $\mathrm{eV} / \Delta$ and $L / \xi_{0}$ for $R=0.16$ and $k T \ll \Delta$; light areas correspond to peaks, dark to dips. $\left(\hbar v_{\mathrm{F}} / E_{\mathrm{A}}=0\right)$. Right: A contour plot of the third derivative $d I^{3} / d V^{3}$ around zero, giving the extremal points of $d I^{2} / d V^{2}$ in the intersections of the dark and white areas. The minima in Eq. (1) are indicated by dashed lines.

predicts dips in the second derivative at voltages $V_{\min }=$ $2 \Delta /$ en independent of junction length 22].

Comparing theory and experiment, we see that the coherent MAR theory (Fig. 3) is able to explain the overall shift in the experimentally observed SGS towards lower voltage for increasing junction length $L$ (see Fig. 2). However, to fit the measured positions of the dips in the second derivative with Eq. (II), one needs to include an additional length $\hbar v_{\mathrm{F}} / E_{\mathrm{A}}$. The best fit, including the SGS to fifth order, is obtained for $\hbar v_{\mathrm{F}} / E_{\mathrm{A}}=0.54 \xi_{0}$ for both junctions on chip A, $0.7 \xi_{0}$ for both junctions on chip B and $0.7 \xi_{0}$ for chip C. We note that the values for the effective lengths $L_{\text {eff }}$ are partly outside the range of validity of Eq. (1), which might introduce some error in the fitted values $\hbar v_{\mathrm{F}} / E_{\mathrm{A}}$. The fact that the junctions on the same chip give the same $E_{\mathrm{A}}$ suggests that $E_{\mathrm{A}}$ is sensitive to the sample preparation.

Although the dip and peak positions of the SGS can be well reproduced by the theory, the theoretically calculated amplitudes (not shown) of the SGS-features are much larger than the ones observed in the experiment. One reason might be residual inelastic scattering or dephasing, not taken into account in the model. Another reason might be that the theory assumes a voltage bias, while the measurements are carried out with current bias, giving rise to voltage fluctuations which smear the SGS.

The experimentally measured excess current $I_{\text {exc }}=$ $I-V / R_{\mathrm{N}}$ at voltages $V \gg \Delta / e$ is presented in the left panel in Fig. 4. As seen from numerics in the right panel of Fig. 4 for both $E_{\mathrm{A}}=1.4 \Delta$ and $1.8 \Delta$, the excess current decreases monotonically with junction length $L$ for all lengths $L<\xi_{0}$, in qualitative agreement with the experiment. However, this decay is slower than the measured one. Moreover, the theoretical excess current exceeds the experimental one. This discrepancy can be attributed to residual inelastic scattering, similar to the suppression of the amplitude of the SGS mentioned 


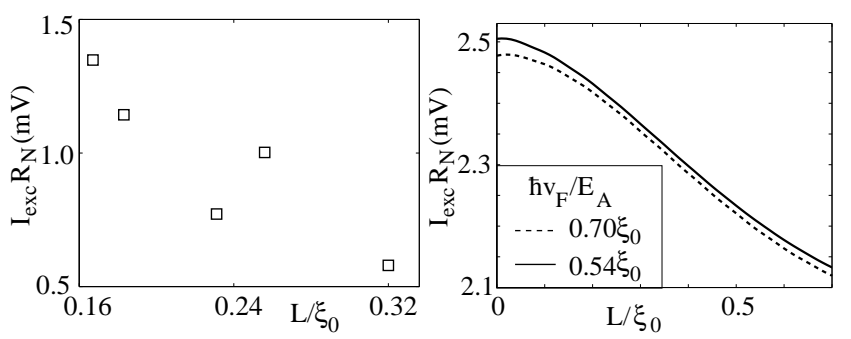

FIG. 4: Left: Excess current at $T=1.8 \mathrm{~K}$ for the five experimentally investigated junctions. Right: Numerically calculated excess current as a function of length $L$, for $R=0.16$ and $k_{B} T \ll \Delta$.

above. The excess current is carried by quasiparticles which traverse the junction one and two times. The single-particle processes give a negative contribution to the excess current while the two-particle processes yield a positive contribution. Since quasiparticles carrying the two-particle current spend longer time in the junction, inelastic scattering leads to a stronger suppression of the two-particle current compared to the one-particle current. This results in an excess current with a stronger length-dependent decay than predicted by the coherent theory.

We also compare the experimentally measured dc Josephson currents with the result of the model. The expression for Josephson current in terms of the normal scattering amplitudes and the Andreev reflection amplitudes is given in Ref. 17]. Using the value of $E_{\mathrm{A}}$ deduced from the SGS fitting, we see in Fig. 1 that the temperature dependence of the experimentally measured critical current is well reproduced by the theory. However, the theoretical overall magnitude is 1.65 times (for $L=105 \mathrm{~nm}$ ) resp. 2.4 times (for $L=160 \mathrm{~nm}$ ) larger than the experimental value. This discrepancy indicates, in line with the discussion for the SGS-amplitude and the excess current above, the presence of some decoherenece mechanism. Note that the discrepancy is much smaller than previously reported [2, 4, 5], because the longer effective junction length reduces the theoretical critical current to a value more similar to the experimental one. The significant part is that this effective length has been determined from a best fitting to the SGS.

In conclusion, we have studied, experimentally and theoretically, coherent current transport in wide ballistic S-2DEG-S junctions. It is found experimentally that both the SGS and the excess current show a systematic dependence on length of the junction. We show that these observations can be qualitatively explained within a coherent theory of MAR. Our investigation also points towards additional mechanisms, e.g. decoherence, to be included in the theory to obtain a quantitative agreement with experiments.

The work was supported by MANEP (P.S.), STINT (A.I.), the Humboldt Foundation, the BMBF and the
ZIP-programme of the German government (G.J.), the Swedish grant agencies TFR (P.S.), SSF (V.S.), VR (G.W., V.S.) and KVA (E.B.), and by the DFG via SFB 508 (R.K., A.R., T.M. and U.M.)

* Present adress: Department of Physics, University of the Western Cape, Cape Town, Bellville 7535, South Africa.

† Present adress: NTT Basic Research Labs., 3-1, Morinosato Wakamiya, Atsugi, Kanagawa, 243-0198, Japan.

[1] I.O. Kulik, Zh. Eksp. Teor. Fiz. 57, 1745 (1969) [Sov. Phys. JETP 30, 944 (1970)].

[2] H. Takayanagi and T. Kawakami, Phys. Rev. Lett. 54, 2449 (1985); H. Takayanagi, T. Akazaki, and J. Nitta, ibid 75, 3533 (1995).

[3] T. Schäpers, Superconductor/Semiconductor junctions, Springer (2001); A. Chrestin et al., Superlatt. Microstr. 25, 711 (1999).

[4] T. Akazaki et al., Appl. Phys. Lett. 68, 418 (1996).

[5] A.F Morpurgo et al., Phys. Rev. Lett. 79, 4010 (1997); G. Bastian et al., ibid 81, 1686 (1998); K.W. Lehnert et al., ibid 82, 1265 (1999); L. Mur et al., Phys. Rev. B. 54, R2327 (1996); A. Chrestin, T. Matsuyama, and U. Merkt, ibid 55, 8457 (1997); J. P. Heida et al., ibid 60, 13135 (1999).

[6] T. Schäpers et al., Appl. Phys. Lett., 71, 3575 (1997); A Richter et al., Supercond. Sci. Technol. 12, 874 (1999).

[7] V.Z. Kresin, Phys. Rev. B. 34, 7587 (1986); U. Schüssler and R. Kümmel, ibid 47, 2754 (1993); A. Chrestin, T. Matsuyama, and U. Merkt, ibid 49, 498 (1994).

[8] T.M. Klapwijk, G.E. Blonder, and M. Tinkham, Physica B+C109-110, 1657 (1982).

[9] M. Octavio et al., Phys. Rev. B. 27, 6739 (1983).

[10] E.N. Bratus', V.S. Shumeiko, and G.Wendin, Phys. Rev. Lett. 74, 2110 (1995); D. Averin and A. Bardas, ibid 75, 1831 (1995); J.C. Cuevas, A. Martin-Rodero, and A.L. Yeyati, Phys. Rev. B. 54, 7366 (1996).

[11] See e.g. E. Scheer et al. Nature 394, 154 (1998); B. Ludoph et al., Phys. Rev. B. 61, 8561 (2000).

[12] G. Johansson et al., Phys. Rev. B 60, 1382 (1999); Superlatt. Microstr. 25, 906, (1999).

[13] Å Ingerman et al., Phys. Rev. B 64, 144504 (2001).

[14] Details of the junction fabrication can be found in A. Chrestin et al., 3].

[15] U. Gunsenheimer and A.D. Zaikin, Phys. Rev. B. 50, 6317 (1994).

[16] S. Datta, Ph. Bagwell and M.P. Anatram, Phys. Low. Dim. Struct. 3, 1 (1996); V.S. Shumeiko, E.N. Bratus, and G. Wendin, Low. Temp. Phys. 23, 181 (1997).

[17] C.W.J. Beenakker, Phys. Rev. Lett 67, 3836 (1991).

[18] A.F. Volkov et al., Physica C 7, 261 (1995).

[19] This gives qualitatively the same result as using the Andreev reflection amplitude of A. A. Golubov and M. Yu. Kupriyanov, JETP Lett. 61, 851 (1995), with a proximity induced gap $E_{\mathrm{g}} \approx\left(1 / \Delta+1 / E_{\mathrm{A}}\right)^{-1}$ in the $2 \mathrm{DEG}$ underneath the superconductor.

[20] This summation effectively averages out all features of the current on the length scale $\lambda_{\mathrm{F}}$.

[21] We focus on the minima in $d^{2} I / d V^{2}$ which, in contrast to the extremal points in $d I / d V$, approach $2 \Delta /$ en for $L \rightarrow 0$ and are essentially independent on $R$ for $R<0.16$. 
[22] U. Zimmermann et al., Z. Phys. B. 97, 59 (1995). 